\section{Dor lombar e transtornos mentais comuns na Estratégia Saúde da Família: uma associação pouco reconhecida}

\author{
Low back pain and common mental disorders in Family Health \\ Strategy: an association little recognized
}

\section{Dolor lumbar y trastornos mentales comunes en la Estrategia Salud de la Familia: una asociación poco reconocida}

\section{Resumo}

Objetivo: Descrever o perfil sociodemográfico dos pacientes atendidos na Estratégia Saúde da Família de quatro capitais brasileiras (Rio de Janeiro, São Paulo, Porto Alegre e Fortaleza) com dor lombar e investigar a associação entre dor lombar e depressão, ansiedade e somatização. Métodos: Estudo de corte transversal com 1857 pacientes atendidos nos anos de 2009 (setembro a novembro) e 2010 (junho a agosto). Instrumentos: rastreio de somatização (SOMS-2), avaliação de ansiedade e depressão (HAD), Questionário Geral do Paciente, para dados sociodemográficos e o Formulário do Profissional Assistente, com as informações referentes as consultas. A dor lombar foi analisada a partir da frequência de três diferentes formas de apresentação: queixa autorreferida pelo paciente, sintoma registrado pelo médico e diagnóstico de lombalgia confirmado pelo médico. Resultados: $77,3 \%$ eram mulheres com mais de quatro anos de estudo e renda per capita inferior a um salário mínimo e meio. Encontramos associação significativa de queixa de dor lombar com ansiedade (OR=1,5, 95\% IC 1,02-2,16) e somatização (OR=1,8, 95\% IC 1,12-2,88), mas não com depressão. Pacientes que apresentavam queixa de dor lombar, porém sem registro do sintoma pelo médico, apresentaram associações ainda mais fortes com ansiedade $(\mathrm{OR}=1,6$, $95 \%$ IC 1,03-2,63) e somatização (OR=2,3, 95\% IC 1,33-3,99). A confirmação do diagnóstico de dor lombar pelo médico não se associou significativamente com nenhum transtorno. Conclusão: Considerando a dor lombar como uma das queixas de maior prevalência na Atenção Primária e sua associação significativa com ansiedade e somatização, recomenda-se a abordagem da ansiedade e somatização em pacientes com queixa de dor lombar.

Palavras-chave: Dor Lombar; Atenção Primária à Saúde; Depressão; Ansiedade; Transtornos Somatoformes

\author{
Janete Alves Araujo ${ }^{a}$ \\ Mônica Rodrigues Campos ${ }^{b}$ \\ Marcos Vinícius Ferreira dos Santos ${ }^{b}$ \\ Daniel Almeida Gonçalves ${ }^{\complement}$ \\ Jair de Jesus Maric \\ Luis Fernando Tófoli ${ }^{d}$ \\ Dinarte Ballester ${ }^{e}$ \\ Sandra Fortes ${ }^{\mathrm{a}}$
}

a Universidade do Estado do Rio de Janeiro (UERJ). Rio de Janeiro, RJ, Brasil. netteallves@hotmail.com (Autora correspondente); sandrafortes@gmail.com

${ }^{\mathrm{b}}$ Fundação Oswaldo Cruz (FIOCRUZ). Rio de Janeiro, RJ, Brasil. monicarodriguescampos@gmail.com; mvsantos@hotmail.com

c Universidade Federal de São Paulo (UNIFESP). São Paulo, SP, Brasil. daniel.almeida.33@gmail.com; jamari17@gmail.com

d Universidade Estadual de Campinas (UNICAMP). Campinas, SP, Brasil. tofoli@fcm.unicamp.br

e Sistema de Saúde Mãe de Deus. Porto Alegre, RS, Brasil. dinarte.ballester@gmail.com
Como citar: Araujo JA, Campos MR, Santos MVF, Gonçalves DA, Mari JJ, Tófoli LF, et al. Dor lombar e transtornos mentais comuns na Estratégia Saúde da Família: uma associação pouco reconhecida. Rev Bras Med Fam Comunidade. 2018;13(40):1-14. http://dx.doi.org/10.5712/rbmfc13(40)1740
Fonte de financiamento:

$\mathrm{O}$ CNPq financiou a pesquisa fonte e a CAPES forneceu bolsa de mestrado para a pesquisa atual.

Parecer CEP:

2257-CEP/HUPE, aprovado em 10/12/2008.

Conflito de interesses:

declaram não haver.

Procedência e revisão por pares:

revisado por pares.

Recebido em: 18/04/2018.

Aprovado em: 17/08/2018. 


\begin{abstract}
Objective: To describe the demographic profile of the patients assisted in the Family Health Strategy of four Brazilian capitals (Rio de Janeiro, São Paulo, Porto Alegre and Fortaleza) with low back pain and investigate the association between low back pain and depression, anxiety and somatization. Methods: Cross-sectional study with 1857 patients served in the years 2009 (September to November) and 2010 (June to August). Instruments: Screening of somatization (SOMS-2), evaluation of anxiety and depression (HAD), General Questionnaire of the Patient, for sociodemographic data and the Form of the Professional Assistant, with the information regarding the consultations. The low back pain was analyzed from the frequency of three different forms of presentation: auto reference complaint by the patient, symptom recorded by the doctor and diagnosis of backache confirmed by the doctor. Results: $\mathbf{7 7 . 3 \%}$ were women with more than four years of study and per capita income less than a minimum wage and a half. We found significant association of low back pain complaints with anxiety $(\mathrm{OR}=1.5,95 \%$ IC 1,02-2,16) and somatization $(\mathrm{OR}=1.8,95 \%$ IC 1,12-2,88) but not with depression. Patients who complained of low back pain, but there was no record of the symptom by the doctor, presented even stronger associations with anxiety $(\mathrm{OR}=1.6,95 \% \mathrm{IC} 1,03-2,63)$ and somatization $(\mathrm{OR}=2.3$, 95\% IC 1,33-3,99). Confirmation of the diagnosis of low back pain by the doctor has not been associated significantly with any disorder. Conclusion: Considering low back pain as one of the most prevalence complaints in Primary Care and its significant association with anxiety and Somatization, it is recommended to approach anxiety and somatization in patients with a complaint of low back pain.
\end{abstract}

Keywords: Low Back Pain; Primary Health Care; Depression; Anxiety; Somatoform Disorders

\title{
Resumen
}

Objetivo: Describir el perfil demográfico de los pacientes asistidos en la Estrategia Salud de la Familia de cuatro capitales brasileño (Rio de Janeiro, São Paulo, Porto Alegre y Fortaleza) con dolor lumbar e investigar la asociación entre el dolor lumbar y la depresión, ansiedad y somatización. Métodos: Estudio transversal con 1857 pacientes atendidos en los años 2009 (septiembre a noviembre) y 2010 (junio a agosto). Instrumentos: seguimiento de la somatización (SOMS-2), evaluación de la ansiedad y depresión (HAD), Cuestionario General del Paciente, para datos sociodemográficas y el Reporte del Profesional Asistente, con la información relativa a las consultas. El dolor lumbar fue analizado a partir de la frecuencia de tres diversas formas de presentación: queja auto-referida por el paciente, síntoma registrado por el médico y diagnosis del lumbago confirmado por el médico. Resultados: $77,3 \%$ fueron mujeres con más de cuatro años de estudio y renta per cápita menos de un salario mínimo y medio. Encontramos la asociación significativa de queja del dolor lumbar con la ansiedad (RM=1,5, el 95\% IC 1,02-2,16) y somatización (RM=1,8, el 95\% IC 1,12-2,88) pero no con la depresión. Los pacientes que se quejaron de dolor lumbar, pero no había registro del síntoma por el médico, presentaron asociaciones incluso más fuertes con la ansiedad $(R M=1,6$, el $95 \%$ IC 1,03-2,63) y somatización ( $R M=2,3$, el 95\% IC 1,33-3,99). La confirmación del diagnóstico de dolor lumbar por el médico no se ha asociado significativamente con ningún trastorno. Conclusión: Considerando el dolor lumbar como una de las quejas prevalentes de la mayoría en la Atención Primaria y su asociación significativa con la ansiedad y la somatización, se recomienda abordar a la ansiedad y a la somatización en pacientes con quejas de dolor lumbar.

Palabras clave: Dolor de la Región Lumbar; Atención Primaria de Salud; Depresión; Ansiedad; Trastornos Somatomorfos

\section{Introdução}

A dor lombar é uma das principais causas mundiais de incapacidade, ${ }^{1}$ com frequência esta associada à comorbidade psiquiátrica e apresenta impacto econômico considerável. ${ }^{2}$ De acordo com a Organização Mundial de Saúde,$^{3}$ a dor lombar afeta mais de $80 \%$ das pessoas em algum momento de sua vida. Destaca-se, portanto, que a dor lombar ainda é um problema importante de saúde pública, acarretando prejuízos para os indivíduos e a sociedade. A prevalência de dor lombar é especialmente importante quando se considera a quantidade de limitação das atividades e de demanda por serviço de saúde que esse problema gera. ${ }^{4}$

Estudos brasileiros ${ }^{5-7}$ destacam a dor lombar como sintoma importante nos atendimentos da Atenção Primária à Saúde (APS), caracterizado como um dos principais motivos da busca por consulta. As queixas somáticas constituem uma das principais justificativas dos usuários pela busca de atendimento, porém só para uma fração dessas queixas encontram-se "explicações orgânicas confiáveis". ${ }^{8}$

Segundo Rosendal et al., ${ }^{9}$ uma em cada três consultas é encerrada sem apresentar um diagnóstico específico e uma em cada seis consultas abarca Sintomas sem Explicação Médica (SEM), "que podem se 
apresentar de forma muito variada: de conjuntos de poucos sintomas autolimitados a padrões crônicos de apresentação", ${ }^{9,10}$ variando em um continuum de intensidade e gravidade. Esses sintomas representam um importante papel na APS, pela sua frequência e necessidade de intervenções específicas.

"As síndromes somatoformes crônicas são os quadros mais estáveis, duradouros e graves de sintomas SEM, sendo definidos pela psiquiatria e por outras especialidades médicas". ${ }^{11}$ Resumindo, como um processo, a manifestação da somatização vai de sintomas leves até quadros mais crônicos tais como o diagnóstico psiquiátrico de transtornos somatoformes, uma categoria do CID 10. Neste artigo utilizamos o SOMS-2 para rastreamento de somatização crônica.

Fatores psicológicos podem influenciar a forma como o sujeito percebe a dor, principalmente a somatização, a depressão e a ansiedade ${ }^{12,13}$ e devem ser considerados como elementos que podem influenciar na causa, na intensidade e continuidade da dor, assim como as intervenções dos profissionais de saúde no cuidado com o paciente que apresenta o sintoma de dor. ${ }^{14}$

Em relação à dor lombar, em que temos uma condição complexa produzida por múltiplos fatores, existem evidências ${ }^{13}$ de que as dificuldades psicossociais e fatores psicológicos podem estar associados à dor lombar. Apesar do reconhecimento de que as queixas de dor estão associadas com problemas psicológicos, a relação exata entre comportamento de dor e fatores psicológicos ainda precisa ser mais estudada. ${ }^{13}$

Portanto, o contexto da APS se caracteriza por um campo substancial para a investigação da associação entre a dor lombar com sofrimento psicológico (depressão, ansiedade e somatização). No Brasil, no âmbito da APS, há poucos trabalhos de investigação sobre a dor lombar, tanto a nível epidemiológico como aos fatores psicológicos relacionados a ela.

A relevância deste trabalho baseia-se na necessidade de estudos que se proponham investigar o perfil sociodemográfico dos pacientes atendidos em unidades de saúde, os sintomas mais relatados e os fatores psicológicos a eles associados. A dor tem sido frequentemente considerada um sintoma que funciona como sinal de alerta referente ao processo de adoecimento do sujeito. Dentro do modelo biomédico, o objetivo da terapêutica reside na identificação do agente causal para direcionar o tratamento e atingir uma resolução para esse problema. No entanto, existe uma complexa interação de fatores (biológicos, psicológicos, sociais) que influenciam na evolução do quadro doloroso, ${ }^{15,16}$ que ainda não está bem estabelecida.

AAPS é porta de entrada prioritária para as redes de saúde do SUS e oferece um cuidado baseado em um atendimento humanizado com foco na integralidade e longitudinalidade. ${ }^{17,18} \mathrm{~A}$ Estratégia Saúde da Família (ESF) tem o objetivo de reorganizar a APS e reorientar o modelo assistencial de saúde. De acordo com o Ministério da Saúde no Caderno de Atenção Básica número 28 (Acolhimento à demanda espontânea - Queixas mais comuns na Atenção Básica), a dor lombar é uma das queixas que podem ser frequentes na APS e em $80 \%$ dos casos não se tem a identificação de uma causa orgânica precisa. ${ }^{17}$

Este artigo é parte da dissertação de mestrado defendida no Programa de Pós-graduação em Ciências Médicas da Universidade Estadual do Rio de Janeiro, que objetivou descrever o perfil sociodemográfico dos pacientes com dor lombar e investigar a associação entre a dor lombar com depressão, ansiedade e somatização em pacientes da Estratégia Saúde da Família de quatro capitais brasileiras (Rio de Janeiro, São Paulo, Fortaleza e Porto Alegre) e, a partir desse conhecimento, contribuir para a discussão do cuidado a estes pacientes dentro de uma visão integral, característica da APS. 


\section{Métodos}

\section{Desenho da pesquisa e população}

Estudo transversal multicêntrico, baseado em pesquisas realizadas em 2009 e 2010 em quatro capitais, que representam diferentes regiões do Brasil com características geográficas e culturais distintas. $O$ estudo com o título "Avaliação de um modelo de capacitação em saúde mental na atenção básica: cuidados integrais na prática do matriciamento" foi originalmente construído com o objetivo de avaliar o impacto das capacitações em saúde mental nas atividades assistenciais na APS, cujos resultados já se encontram publicados. ${ }^{19}$

O estudo foi realizado em duas etapas, com um desenho pré-pós quasi-experimental, de setembro a novembro de 2009 e de junho a agosto de 2010. Para o presente estudo, foram utilizados os dados referentes às duas etapas da pesquisa original. A população de interesse foi composta por pacientes de 20 unidades da ESF, atendidos por 27 equipes de saúde (médicos e enfermeiros), essas unidades foram escolhidas pelas Secretarias Municipais de Saúde onde a formação em saúde mental na atenção primária foi declarada uma prioridade.

O número de equipes avaliadas em cada cidade foi proporcional ao número de profissionais treinados em cada uma delas: havia 3 equipes em Porto Alegre, 3 em Fortaleza, 6 em São Paulo e 12 no Rio de Janeiro. Todos os pacientes com idades entre 18 e 65 anos, e médicos e enfermeiras foram convidados a participar da pesquisa. Mulheres grávidas e pacientes com déficit cognitivo não foram incluídos. Nenhum paciente que participou da primeira etapa (1260 pacientes) foi autorizado a participar da segunda (597 pacientes). ${ }^{19,20}$ Para este estudo, a amostra foi coletada e analisada em conjunto.

\section{Coleta de dados}

$\mathrm{Na}$ sala de espera das unidades de saúde, até se obter um número mínimo de 30 pacientes por profissional, todos os pacientes aguardando atendimento médico ou de enfermagem foram abordados por um pesquisador que informou sobre os objetivos e os procedimentos da pesquisa, obteve o consentimento por escrito dos pacientes, e aplicou a versão em português do General Health Questionnaire (GHQ-12), ${ }^{21,22}$ da Hospital Anxiety and Depression Scale (HAD), ${ }^{23}$ do Screening for Somatoform Symptoms (SOMS-2 $)^{24}$ e um questionário sociodemográfico.

Estes instrumentos foram lidos para os pacientes, adaptação metodológica que tem sido utilizada em pesquisas na rede pública brasileira ${ }^{21,25-27}$ em virtude da baixa escolaridade média da população. $O$ Formulário do Profissional Assistente foi preenchido pelos médicos e enfermeiras que participaram das capacitações, ao final de cada consulta, e entregue ao pesquisador. A coleta de dados foi supervisionada em cada unidade por um psicólogo de formação, com quatro a cinco aplicadores. Para a entrada dos dados foi utilizada máscara eletrônica (Access), processo de dupla digitação e Excel. A descrição detalhada dos procedimentos de coleta de dados encontra-se em dois artigos publicados anteriormente. ${ }^{19,20}$

\section{Procedimentos analíticos}

O Questionário Geral foi utilizado para as variáveis socioeconômicas e demográficas que foram codificadas de forma dicotômica, em virtude da homogeneidade da amostra, ${ }^{19}$ ou seja, devido a um 
pequeno número de participantes em algumas categorias das variáveis. Foram selecionadas as variáveis: idade (variável categórica), sexo, etnia autorreferida, estado civil, escolaridade e renda per capita mensal (Salário Mínimo 2009: R\$ 465,00 e 2010: R\$ 510,00). No mesmo questionário a pergunta "Que sintomas ou problemas o trazem ao médico?" Foi utilizada para a construção da variável: "queixa de dor lombar autorreferida pelo paciente".

O Formulário do Profissional Assistente foi utilizado para a construção das variáveis: "Sintoma registrado pelo médico" e "Diagnóstico de lombalgia confirmado" (pelo médico de acordo com o CID 10).

$\mathrm{Na}$ análise do HAD, foi considerado para casos sugestivos de depressão e ansiedade o ponto de corte de até oito pontos como negativo e a partir de nove pontos como positivo, conforme previamente validado no Brasil. ${ }^{23}$ A presença de somatização crônica, a partir do instrumento SOMS-2, foi considerada positiva quando os seguintes critérios estavam presentes: quatro ou mais queixas físicas sem um diagnóstico médico em um período de dois ano ${ }^{28}$ apresentando algum grau de incapacidade e que tenham afetado o bem-estar.

Os dados foram analisados no programa Statistical Package for the Social Science - SPSS 17 (SPSS Inc., Chicago, IL, USA). Inicialmente, realizou-se a estatística descritiva, visando calcular a frequência referente às variáveis sociodemográficas e as variáveis de dor lombar. Em seguida, as frequências foram tabuladas para a caracterização do perfil sociodemográfico dos pacientes com dor lombar e para avaliação da associação entre dor lombar e a situação de saúde mental (ansiedade, depressão e somatização).

Foram calculados Odds ratio (OR) com IC de 95\% para estimar a associação entre as variáveis de dor lombar ("queixa autorreferida pelo paciente", "sintoma registrado pelo médico" e "diagnóstico de lombalgia confirmado pelo médico") e a presença de sofrimento psicológico ("depressão provável”, "ansiedade provável" e somatização). Foram realizados teste do Qui-quadrado $\left(\mathrm{X}^{2}\right)$, com um valor-p de $5 \%$ para avaliar a associação existente entre as variáveis e comparar as divergências entre as frequências observadas e esperadas. Foi também calculado o OR de Mantel-Haenszel e estratificado por município, bem como respectivos IC 95\% e p-valor, para todos os desfechos sob investigação, considerando as exposições de saúde mental (depressão, ansiedade e somatização).

\section{Aspectos éticos e financiamento}

O protocolo de pesquisa original foi aprovado pelo Comitê de Ética em Pesquisa da Universidade Federal de São Paulo e Universidade do Estado do Rio de Janeiro e todos os quatro municípios permitiram a investigação em seus centros de saúde da Atenção Primária. Encontra-se devidamente submetida e aprovada pelo Comitê de Ética em Pesquisa do Hospital Universitário Pedro Ernesto, sob o número № 2257-CEP/HUPE, bem como pelo Comitê de Ética em Pesquisa da Secretaria Municipal de Saúde e Defesa Civil do Rio de Janeiro, sob número № 34/09 CAAE no: 0031.0.314.000-09.

A pesquisa fonte ("Avaliação de um modelo de capacitação em saúde mental na atenção básica: cuidados integrais na prática do matriciamento") foi financiada pelo CNPq e a CAPES forneceu bolsa de mestrado para o Programa de Pós-Graduação em Ciências Médicas (PGCM/UERJ) para o desenvolvimento da pesquisa atual. 


\section{Resultados}

\section{Dados sociodemográficos e prevalência de dor lombar}

No total de 1857 pacientes, encontramos uma distribuição com maioria de mulheres $(77,3 \%)$ com mais de 35 anos de idade e mais de quatro anos de estudo, sendo a maioria casadas, de etnia autorrelatada como não branca. A quase totalidade dos pacientes declarou uma renda per capita inferior a um salário-mínimo e meio na época, ou seja, no ano de 2009 menos que $R \$ 697,50$ e no ano de 2010 menos que $R \$ 765,00$, conforme Tabela 1. A prevalência de pacientes com dor lombar foi de $118(7 \%)$ para queixa autorreferida pelo paciente, de 85 (5\%) para o sintoma registrado pelo médico e $36(2 \%)$ para o diagnóstico de lombalgia confirmado pelo médico.

Tabela 1. Características sociodemográficas dos pacientes entrevistados. Rio de Janeiro, São Paulo, Fortaleza e Porto Alegre, 2009/2010.

\begin{tabular}{|c|c|c|c|c|}
\hline \multirow[t]{2}{*}{ Características } & $\begin{array}{l}\text { Amostra } \\
\text { Total (\%) }\end{array}$ & $\begin{array}{c}\text { Queixa de dor lombar } \\
\text { autorreferida pelo } \\
\text { paciente (\%) }\end{array}$ & $\begin{array}{c}\text { Sintoma de dor lombar } \\
\text { registrado pelo médico } \\
(\%)\end{array}$ & $\begin{array}{l}\text { Diagnóstico de } \\
\text { lombalgia confirmado } \\
\text { pelo médico }(\%)\end{array}$ \\
\hline & $(\mathrm{N}=1857)$ & $(\mathrm{N}=118)$ & $(\mathrm{N}=85)$ & $(\mathrm{N}=36)$ \\
\hline \multicolumn{5}{|l|}{ Gênero } \\
\hline Feminino & 77,3 & 78,0 & 71,8 & 66,6 \\
\hline \multicolumn{5}{|c|}{ Faixa etária (anos) } \\
\hline$>35$ & 67,7 & 73,8 & 69,4 & 69,5 \\
\hline \multicolumn{5}{|c|}{ Renda per capita mensal } \\
\hline$<1,5 \mathrm{SM}$ & 86,0 & 86,4 & 87,1 & 86,1 \\
\hline \multicolumn{5}{|l|}{ Cor } \\
\hline Não branca & 70,8 & 69,5 & 64,7 & 63,8 \\
\hline \multicolumn{5}{|l|}{ Estado civil } \\
\hline Casados/União & 61,1 & 61,9 & 68,2 & 66,6 \\
\hline \multicolumn{5}{|c|}{ Nível de escolaridade (anos) } \\
\hline 4 ou mais & 67,4 & 69,5 & 62,4 & 63,8 \\
\hline
\end{tabular}

\section{Associações de dor lombar com depressão, ansiedade e somatização}

Os dados da Tabela 2 mostram as associações entre a queixa autorreferida de dor lombar e sofrimento psicológico (depressão, ansiedade e somatização) em que se verificou uma associação significativa com ansiedade (OR=1,49; IC 95\% 1,02 a 2,16) e somatização (OR=1,80; IC 95\% 1,12 a 2,88), mas não com depressão (OR=1,00; IC 95\% 0,66 a 1,55).

Ao analisarmos a Tabela 3, constatamos que não foi observada nenhuma associação significativa entre o sintoma de dor lombar registrado pelo médico, com depressão (OR=0,79; IC 95\% 0,46 a 1,34) ansiedade (OR=1,36; IC 95\% 0,88 a 2,11) ou somatização (OR=1,11; IC 95\% 0,59 a 2,08). Da mesma forma, em relação ao diagnóstico de lombalgia confirmado pelo médico, também não foram verificadas associações significativas com depressão (OR=0,84; IC 95\% 0,38 a 1,86), ansiedade (OR=1,68; IC 95\% 0,87 a 3,25) ou somatização (OR=1,64; IC 95\% 0,71 a 3,80), conforme mostra a Tabela 4. 
Tabela 2. Associação entre ter queixa de dor lombar autorreferida pelo paciente e os desfechos em saúde mental: ansiedade, depressão e somatização. Rio de Janeiro, São Paulo, Fortaleza e Porto Alegre, 2009/2010.

\begin{tabular}{|c|c|c|c|c|c|c|c|c|}
\hline \multirow{3}{*}{$\begin{array}{l}\text { Desfechos em } \\
\text { saúde Mental }\end{array}$} & \multicolumn{4}{|c|}{ Queixa de dor lombar autorreferida pelo paciente } & \multirow{2}{*}{\multicolumn{2}{|c|}{ Total }} & \multirow{2}{*}{\multicolumn{2}{|c|}{ Associação Estatística }} \\
\hline & \multicolumn{2}{|c|}{ Não } & \multicolumn{2}{|c|}{ Sim } & & & & \\
\hline & $\mathbf{n}$ & $\%$ & $\mathbf{n}$ & $\%$ & $\mathbf{N}$ & $\%$ & $\begin{array}{l}\text { OR bruto (IC } \\
95 \% \text { ) p-valor }\end{array}$ & $\begin{array}{l}\text { OR de } \mathrm{MH}^{*} \text { (IC } \\
95 \% \text { ) p-valor }\end{array}$ \\
\hline \multicolumn{9}{|c|}{ Depressão ( $\mathrm{N}=1857)$} \\
\hline Não & 1301 & 75,0 & 88 & 75,0 & 1389 & 75,0 & 1,00 & 1,02 \\
\hline Sim & 438 & 25,0 & 30 & 25,0 & 468 & 25,0 & $(0,66-1,55)$ & $(0,67-1,57)$ \\
\hline Total & 1739 & 100,0 & 118 & 100,0 & 1857 & 100,0 & 0,954 & 0,920 \\
\hline \multicolumn{9}{|c|}{ Ansiedade ( $\mathrm{N}=1857)$} \\
\hline Não & 1096 & 63,0 & 63 & 53,0 & 1389 & 62,0 & 1,49 & 1,48 \\
\hline Sim & 643 & 37,0 & 55 & 47,0 & 468 & 38,0 & $(1,02-2,16)$ & $(1,01-2,15)$ \\
\hline Total & 1739 & 100,0 & 118 & 100,0 & 1857 & 100,0 & 0,037 & 0,043 \\
\hline \multicolumn{9}{|c|}{ Somatização ( $N=1857)$} \\
\hline Não & 1523 & 88,0 & 94 & 80,0 & 1617 & 87,0 & 1,80 & 1,71 \\
\hline Sim & 216 & 12,0 & 24 & 20,0 & 240 & 13,0 & $(1,12-2,88)$ & $(1,07-2,76)$ \\
\hline Total & 1739 & 100,0 & 118 & 100,0 & 1857 & 100,0 & 0,013 & 0,026 \\
\hline
\end{tabular}

* OR de MH: Odds Ratio de Mantel-Haenszel.

Tabela 3. Associação entre ter sintoma de dor lombar registrado pelo médico e os desfechos em Saúde Mental: ansiedade, depressão e somatização. Rio de Janeiro, São Paulo, Fortaleza e Porto Alegre, 2009/2010.

\begin{tabular}{|c|c|c|c|c|c|c|c|c|}
\hline \multirow{3}{*}{$\begin{array}{l}\text { Desfechos em Saúde } \\
\text { Mental }\end{array}$} & \multicolumn{4}{|c|}{ Sintoma de dor lombar Registrado pelo médico } & \multirow{2}{*}{\multicolumn{2}{|c|}{ Total }} & \multirow{2}{*}{\multicolumn{2}{|c|}{ Associação Estatística }} \\
\hline & \multicolumn{2}{|c|}{ Não } & \multicolumn{2}{|c|}{ Sim } & & & & \\
\hline & $\mathbf{n}$ & $\%$ & $\mathbf{n}$ & $\%$ & $\mathbf{n}$ & $\%$ & $\begin{array}{l}\text { OR bruto (IC } \\
95 \% \text { ) p-valor }\end{array}$ & $\begin{array}{l}\text { OR de } \mathrm{MH}^{\star} \text { (IC } \\
95 \%) \text { p-valor }\end{array}$ \\
\hline \multicolumn{9}{|l|}{ Depressão ( $\mathrm{N}=1857)$} \\
\hline Não & 1322 & 75,0 & 67 & 79,0 & 1389 & 75,0 & 0,79 & 0,82 \\
\hline Sim & 450 & 25,0 & 18 & 21,0 & 468 & 25,0 & $(0,46-1,34)$ & $(0,48-1,4)$ \\
\hline Total & 1772 & 100,0 & 85 & 100,0 & 1857 & 100,0 & 0,382 & 0,478 \\
\hline \multicolumn{9}{|l|}{ Ansiedade $(\mathrm{N}=1857)$} \\
\hline Não & 1112 & 63,0 & 47 & 55,0 & 1159 & 62,0 & 1,36 & 1,37 \\
\hline Sim & 660 & 37,0 & 38 & 45,0 & 698 & 38,0 & $(0,88-2,11)$ & $(0,88-2,13)$ \\
\hline Total & 1772 & 100,0 & 85 & 100,0 & 1857 & 100,0 & 0,165 & 0,164 \\
\hline \multicolumn{9}{|l|}{ Somatização ( $N=1857)$} \\
\hline Não & 1544 & 87,0 & 73 & 86,0 & 1617 & 87,0 & 1,11 & 1,02 \\
\hline Sim & 228 & 13,0 & 12 & 14,0 & 249 & 13,0 & $(0,59-2,08)$ & $(0,54-1,94)$ \\
\hline Total & 1772 & 100,0 & 85 & 100,0 & 1857 & 100,0 & 0,737 & 0,942 \\
\hline
\end{tabular}

* OR de MH: Odds Ratio de Mantel-Haenszel.

$\mathrm{Na}$ Tabela 5, a análise estatística dos dados mostra que pacientes com 'queixa autorreferida de dor lombar', que não tiveram o sintoma de dor lombar registrado pelo médico, apresentaram uma associação ainda mais forte, com ansiedade (OR=1,65; IC95\% 1,03-2,63) e com somatização (OR=2,30; IC95\% 1,33 a 3,99), mas não com depressão (OR=1,38; IC95\% 0,83 a 2,29). 
Tabela 4. Associação entre ter diagnóstico de lombalgia confirmado pelo médico e os desfechos em Saúde Mental: ansiedade, depressão e somatização. Rio de Janeiro, São Paulo, Fortaleza e Porto Alegre, 2009/2010.

\begin{tabular}{|c|c|c|c|c|c|c|c|c|}
\hline \multirow{3}{*}{$\begin{array}{l}\text { Desfechos em Saúde } \\
\text { Mental }\end{array}$} & \multicolumn{4}{|c|}{ Diagnóstico de lombalgia confirmado pelo médico } & \multirow{2}{*}{\multicolumn{2}{|c|}{ Total }} & \multirow{2}{*}{\multicolumn{2}{|c|}{ Associação Estatística }} \\
\hline & \multicolumn{2}{|c|}{ Não } & \multicolumn{2}{|c|}{ Sim } & & & & \\
\hline & $\mathbf{n}$ & $\%$ & $\mathbf{n}$ & $\%$ & $\mathbf{n}$ & $\%$ & $\begin{array}{l}\text { OR bruto (IC } \\
95 \% \text { ) p-valor }\end{array}$ & $\begin{array}{l}\text { OR de } \mathrm{MH}^{\star} \text { (IC } \\
95 \%) \text { p-valor }\end{array}$ \\
\hline \multicolumn{9}{|l|}{ Depressão ( $\mathrm{N}=1857)$} \\
\hline Não & 1361 & 75,0 & 28 & 75,0 & 1389 & 75,0 & 0,84 & 0,87 \\
\hline Sim & 460 & 25,0 & 8 & 25,0 & 468 & 25,0 & $(0,38-1,86)$ & $(0,4-1,92)$ \\
\hline Total & 1821 & 100,0 & 36 & 100,0 & 1857 & 100,0 & 0,678 & 0,733 \\
\hline \multicolumn{9}{|l|}{ Ansiedade ( $\mathrm{N}=1857)$} \\
\hline Não & 1141 & 63,0 & 18 & 50,0 & 1159 & 62,0 & 1,68 & 1,64 \\
\hline Sim & 680 & 37,0 & 18 & 50,0 & 698 & 38,0 & $(0,87-3,25)$ & $(0,84-3,19)$ \\
\hline Total & 1821 & 100,0 & 36 & 100,0 & 1857 & 100,0 & 0,120 & 0,145 \\
\hline \multicolumn{9}{|l|}{ Somatização (N=1857) } \\
\hline Não & 1588 & 88,0 & 29 & 80,0 & 1617 & 87,0 & 1,64 & 1,42 \\
\hline Sim & 233 & 12,0 & 7 & 20,0 & 240 & 13,0 & $(0,71-3,80)$ & $(0,6-3,33)$ \\
\hline Total & 1821 & 100,0 & 36 & 100,0 & 1857 & 100,0 & 0,645 & 0,425 \\
\hline
\end{tabular}

* OR de MH: Odds Ratio de Mantel-Haenszel.

Tabela 5. Associação entre ter Queixa de dor lombar autorreferida e não ter Sintoma registrado pelo médico (uma subamostra) e os desfechos em saúde Mental: ansiedade, depressão e somatização. Rio de Janeiro, São Paulo, Fortaleza e Porto Alegre, 2009/2010.

\begin{tabular}{|c|c|c|c|c|c|c|c|c|}
\hline \multirow{2}{*}{$\begin{array}{l}\text { Desfecho em Saúde } \\
\text { Mental }\end{array}$} & \multicolumn{4}{|c|}{$\begin{array}{c}\text { Queixa de dor lombar auto referida sem Sintoma } \\
\text { registrado pelo médico }\end{array}$} & \multirow{2}{*}{\multicolumn{2}{|c|}{ Total }} & \multirow{2}{*}{\multicolumn{2}{|c|}{ Associação Estatística }} \\
\hline & \multicolumn{2}{|c|}{ Não } & \multicolumn{2}{|c|}{ Sim } & & & & \\
\hline \multicolumn{9}{|l|}{ Depressão (N=1857) } \\
\hline Sim & 445 & 25,0 & 23 & 32,0 & 468 & 25,0 & $(0,83-2,29)$ & $(0,83-2,27)$ \\
\hline Total & 1784 & 100,0 & 73 & 100,0 & 1857 & 100,0 & 0,200 & 0,222 \\
\hline \multicolumn{9}{|l|}{ Ansiedade ( $\mathrm{N}=1857)$} \\
\hline Não & 1122 & 63,0 & 37 & 51,0 & 1159 & 62,0 & 1,65 & 1,63 \\
\hline Sim & 662 & 37,0 & 36 & 49,0 & 698 & 38,0 & $(1,03-2,63)$ & $(1,02-2,61)$ \\
\hline Sim & 222 & 12,0 & 18 & 25,0 & 240 & 13,0 & $(1,33-3,99)$ & $(1,3-3,97)$ \\
\hline Total & 1784 & 100,0 & 73 & 100,0 & 1857 & 100,0 & 0,000 & 0,004 \\
\hline
\end{tabular}

* OR de MH: Odds Ratio de Mantel-Haenszel. 


\section{DISCUSSÃO}

\section{Resumo dos principais achados do estudo}

No presente estudo, foi encontrada uma prevalência de dor lombar abaixo dos valores encontrados em outros estudos no Brasil e fora do Brasil. Os dados apresentados evidenciaram uma associação estatisticamente significativa entre "queixa autorreferida pelo paciente de dor lombar", com ansiedade e somatização, mas não com depressão. Pacientes que apresentavam queixa de dor lombar, mas sem o registro do sintoma pelo médico, apresentaram associações ainda mais fortes com ansiedade e somatização.

\section{Fortalezas e limitações do estudo}

Por ser um estudo de temporalidade transversal, a maior limitação deste estudo está ligada à impossibilidade de estabelecer relações causais entre as variáveis e investigar a cronificação de dores agudas. Por não ter sido realizada a análise de regressão logística, as interpretações ficaram limitadas. $O$ banco de dados utilizado não foi planejado especialmente para o estudo de dor lombar, portanto, não foi possível o conhecimento da etiologia da dor. Os resultados apresentados neste artigo são consistentes com estudos encontrados na literatura ${ }^{29-31}$ em que transtornos de ansiedade e somatização foram mais prevalentes que transtornos depressivos em pacientes com dor lombar. O presente estudo vem reforçar a associação entre os processos psicológicos e dor lombar, e sua importância na APS.

\section{Comparação com a literatura já existente}

Mirzamani-Bafghi et al. ${ }^{32}$ também demonstraram em seu estudo que o problema psicológico mais presente nos pacientes pesquisados com dor lombar foi a ansiedade, e que ela pode ter desempenhado um papel no início, gravidade e exacerbação ou manutenção da dor. Segundo Reme et al., ${ }^{31}$ a dor lombar e as consequências incapacitantes da ansiedade são tão graves como a depressão e "sugere que a triagem de transtornos de ansiedade pode ser tão importante quanto da depressão em pacientes com dor lombar". ${ }^{32}$

Na literatura, encontramos vários estudos sobre as contribuições dos sintomas de depressão na dor lombar, conforme revisão sistemática e meta-análise realizada recentemente. ${ }^{33}$ Entretanto, a ansiedade tem recebido uma atenção reduzida como uma comorbidade em pacientes com dor lombar. ${ }^{31}$

Nos manuais de orientação do Ministério da Saúde (MS) para a APS, ${ }^{17}$ os profissionais de saúde, ao atenderem usuários com queixa de dor lombar, são orientados a caracterizar a dor, identificar sintomas neurológicos e realizar uma anamnese adequada. Fornecem orientação para o diagnóstico diferencial, sendo que a depressão e a somatização estão presentes entre os sinais de alerta apresentados para o diagnóstico diferencial, porém a ansiedade não é considerada. De acordo com o estudo atual e outros estudos já citados, a ansiedade pode desempenhar um papel importante em pacientes que apresentam dor lombar, portanto, a avaliação de sintomas ansiosos pode contribuir para melhor avaliação e tratamento. 
Em relação à prevalência, encontramos os seguintes resultados de dor lombar: $7 \%$ para queixa autorreferida pelo paciente, $5 \%$ para sintoma registrado pelo médico e $2 \%$ para o diagnóstico de lombalgia confirmado pelo médico. Esses percentuais estão abaixo dos encontrados em estudos na atenção primária fora do Brasil, ${ }^{34,35}$ que encontraram prevalências bem maiores, de $49 \%$ e $64,7 \%$.

Em um estudo brasileiro ${ }^{5}$ em comunidade atendida por unidade de atenção primária à saúde com objetivo de investigar a prevalência de dor crônica em uma cidade no estado do Maranhão, o quadro mais prevalente entre os quadros de dor musculoesquelética foi o de dor lombar, com o percentual de $5,12 \%$ de todos os indivíduos avaliados. Outro estudo brasileiro ${ }^{6}$ realizado em Fortaleza (Ceará) em uma Unidade de Saúde da Família mostrou que a queixa de dor lombar foi a mais referida pelos entrevistados, com um percentual de 13,8\%, valores acima do encontrado no estudo atual. O baixo registro de queixas de dor lombar na pesquisa atual pode indicar uma não valorização dessas queixas por parte dos profissionais de saúde.

\section{Implicações para a pesquisa na área e para a prática dos profissionais}

Segundo o Ministério da Saúde no Caderno de Atenção Básica número 28 (acolhimento à demanda espontânea - Queixas mais comuns na Atenção Básica), somente em $20 \%$ dos casos de dor lombar são encontradas afecções orgânicas que a justifique. ${ }^{17}$ Essas sensações físicas anormais, que podem estar relacionadas a alterações somáticas ou a doenças orgânicas, também estão geralmente associadas a transtornos mentais, ou seja, a sofrimento psíquico inespecífico.

"As doenças são catalogadas em uma sorte de classificação hierárquica, correspondendo à gravidade das lesões anatomopatológicas", portanto, parece que a percepção dos médicos em relação aos sintomas apresentados pelos pacientes tem "pontos cegos" que estão relacionados aos fatores psicológicos e sociais, privilegiando os biológicos. Esse fato ocorre em função do modelo da sua formação. ${ }^{36}$ Esses "pontos cegos" podem justificar a diferença encontrada no estudo atual entre a queixa do paciente de dor lombar antes da consulta com o médico e o registro de dor lombar durante a consulta médica.

Cuidar desses casos é uma função, para a qual, "a formação em medicina ainda não está preparada". 8 A identificação, o registro e o cuidado dessas queixas são uma tarefa que representa um desafio para os profissionais de saúde da APS, e para os usuários, a não identificação intensifica o sofrimento mental. ${ }^{8}$

A relação médico-paciente é uma interação que pode ser complicada, muitas vezes o paciente não consegue expressar suas queixas durante a consulta e os médicos não conseguem reconhecer a realidade do paciente e, apesar da tentativa de ambas as partes, não se consegue um resultado positivo. ${ }^{37} \mathrm{Nem} o$ paciente consegue referir de forma clara seus sintomas, nem o médico consegue perceber ou entender, a "história" que o paciente almeja contar. No presente estudo encontramos em uma subamostra a presença de associações mais fortes de queixa de dor lombar com ansiedade e somatização, nos casos em que houve uma queixa autorreferida pelos usuários na entrevista com o pesquisador, mas não houve registro do sintoma pelo médico na consulta médica, que ocorreu após a entrevista. Portanto, parece que existem sintomas que não são declarados pelos pacientes aos médicos ou não são identificados, e que esses sintomas podem estar relacionados com quadro de sofrimento emocional. 
Existe uma "série significativa de patologias atribuídas à influência exercida sobre o corpo pelas emoções", ${ }^{36}$ e não é fácil fazer o diagnóstico quando o paciente apresenta queixas sem uma etiologia orgânica clara. São pacientes que se consideram doentes fisicamente, porém, em uma parcela significativa dos casos, apresentam problemas psicológicos que influenciam de forma significativa na causa dos seus sintomas. ${ }^{36}$

Nas consultas, espaço considerado com uma dinâmica interpessoal complexa, onde se desenvolve a relação médico e paciente, acontece o encontro de culturas variadas e grupos sociais diferentes, sendo fundamental o desenvolvimento de outros recursos na tentativa de lidar de forma mais eficaz com os usuários que apresentam esses sintomas vagos e difusos. ${ }^{38}$ É essencial que se considere o usuário dentro do seu contexto, e não só a enfermidade que o acomete, visando uma Clínica ampliada, ou seja, que ultrapasse os procedimentos técnicos padronizados. ${ }^{39}$

As origens psíquicas das queixas físicas não podem ser abordadas a partir de diagnósticos de exclusão, ou seja, apenas pela eliminação de outras causas possíveis para os sintomas do paciente. Devem ser abordadas dentro do modelo biopsicossocial, considerando todos os aspectos inerentes ao espaço vital do paciente (orgânico, psicológico, cultural e socioeconômico) em que cada aspecto influencia e é influenciado de modo dinâmico. ${ }^{40}$ Sendo assim, esses pacientes, na verdade, podem ter um diagnóstico de transtorno mental, ainda não investigado.

A dor lombar afeta funções físicas, interação social e os aspectos psicológicos dos pacientes. É uma questão de saúde pública, sendo relevante a inserção de propostas, tanto para inclusão de programas de treinamento e educação para os profissionais da APS como de programas de ações educativas para os pacientes, com o objetivo de redução da dor e do impacto negativo que causa na funcionalidade dos mesmos. Entre essas propostas, temos a "Back School" (Escola Postural-EP), que, segundo estudos realizados na APS, ${ }^{41-43}$ mostra efeitos positivos, como a melhora da capacidade funcional e qualidade de vida, e a diminuição gradativa da intensidade de dor, em pacientes com dor lombar que participaram dos programas de EP. ${ }^{41}$

Recente estudo publicado pela revista científica The Lancet ${ }^{44}$ (uma das mais antigas revistas sobre medicina e das mais prestigiadas), com uma série de três artigos de autores internacionais liderados pela Prof ${ }^{a}$ Rachelle Buchbinder, Universidade Monash, Melbourne, Austrália, abordam questões sobre a dor lombar e chamam a atenção para a necessidade do reconhecimento mundial das disfunções causadas por esse sintoma e da retirada de práticas prejudiciais.

O primeiro artigo $^{45}$ destaca a complexidade dos fatores que influenciam a dor lombar, tais como fatores psicológicos, sociais e biológicos, e principalmente as dificuldades enfrentadas pelos países de baixa e média renda. $\mathrm{O}$ segundo artigo $^{46}$ relata as propostas para o tratamento e a carência de estudos a respeito da prevenção da dor lombar. O último artigo ${ }^{47}$ convoca a uma ação mais efetiva, destacando a importância do reconhecimento das dificuldades provocadas pela dor lombar e que não é possível a separação dos fatores sociais e econômicos e das crenças pessoais e culturais, que exercem influência sobre ela.

Existe entre os profissionais da APS uma grande dificuldade em cuidar dos pacientes com "problemas de saúde mental", ${ }^{48}$ apesar da ocorrência considerável de casos de usuários com os designados transtornos 
mentais comuns (TMC). Estes procuram uma unidade de atendimento com o objetivo de minimizar a sua dor, que é manifestada por meio de sintomas vagos e difusos. Frequentemente, a esses usuários são conferidos rótulos, como por exemplo: pitiáticos ou poliqueixosos, ${ }^{49}$ e o cuidado adequado de seu sofrimento emocional fica prejudicado.

Uma sugestão que pode melhorar a formação dos profissionais na APS e reduzir essa dificuldade é promover mais capacitações no Método Clínico Centrado na Pessoa (MCCP) ${ }^{50}$ método reconhecido pelo seu amplo potencial de aplicação pelos profissionais de saúde, independentemente da sua formação. Cada vez mais a abordagem centrada na pessoa está ganhando reconhecimento importante como parâmetro de qualidade de assistência. Stewart et al. ${ }^{50}$ apontam que, "quando comparada a modelos tradicionais, a abordagem centrada na pessoa apresenta mais resultados positivos".

A integração do cuidado em Saúde Mental na prática da APS tem sido "considerado um recurso estratégico para trabalhar com pessoas em sofrimento psíquico, em virtude da proximidade e do vínculo que é construído com as famílias e comunidades, o que facilita as intervenções do ponto de vista terapêutico." 51 Os pacientes com queixas físicas inespecíficas, em que se destaca a queixa de dor lombar, constituem um subgrupo particularmente importante dentro destes pacientes.

Os achados mostram que as questões de Saúde Mental estão imbricadas nas queixas relatadas pelos usuários, porém ainda pouca reconhecida e tratada.

Recomenda-se que outros estudos investiguem as relações das queixas relatadas pelos pacientes na APS, com os fatores psicossociais. A importância de mais estudos nessa área está relacionada à necessidade de proteger os pacientes de iatrogenias e pseudodiagnósticos estigmatizantes e de poder fornecer um tratamento mais eficaz e resolutivo.

\section{Referências}

1. Global Burden of Disease Study 2013 Collaborators. Global, regional, and national incidence, prevalence, and years lived with disability for 301 acute and chronic diseases and injuries in 188 countries, 1990-2013: a systematic analysis for the Global Burden of Disease Study 2013. Lancet. 2015;386(9995):743-800. http://dx.doi.org/10.1016/S0140-6736(15)60692-4

2. Gore M, Sadosky A, Stacey BR, Tai KS, Leslie D. The burden of chronic low back pain: clinical comorbidities, treatment patterns, and health care costs in usual care settings. Spine (Phila Pa 1976).2012;37(11):E668-77.http://dx.doi.org/10.1097/BRS.0b013e318241e5de

3. World Health Organization - WHO. Chronic diseases and health promotion. Geneva: WHO; 2011. [acesso 2017 Mar 10]. Disponível em: http://www.who.int/chp/topics/rheumatic/en/

4. Mata MS, Costa FA, Souza TO, Mata ANS, Pontes JF. Dor e funcionalidade na atenção básica à saúde. Ciênc Saúde Coletiva. 2011;16(1):221-30. http://dx.doi.org/10.1590/S1413-81232011000100025

5. Cordeiro Q, El Khouri M, Ota D, Ciampi D, Corbett CE. Lombalgia e cefaleia como aspectos importantes da dor crônica na atenção primária à saúde em uma comunidade da região amazônica brasileira. Acta Fisiatr. 2008;15(2):101-5.

6. Pimentel IRS, Coelho BC, Lima JC, Ribeiro FG, Sampaio FPC, Pinheiro RP, et al. Caracterização da demanda em uma Unidade de Saúde da Família. Rev Bras Med Fam Comunidade. 2011;6(20):175-81.

7. Vieira A, Nonnenmacher LFQ, Bartz PT, Bueno AF, Macedo DS. Perfil de usuários com dores musculoesqueléticas crônicas encaminhadas ao "grupo da coluna". Rev Baiana Saúde Pública. 2014;38(3):571-84. http://dx.doi.org/10.5327/Z0100-0233-2014380300006

8. Tófoli LF, Fortes S, Gonçalves DA, Chazan LF, Ballester D. Somatização e sintomas físicos inexplicáveis para o médico de família e comunidade. Programa de Atualização em Medicina de Família e Comunidade PROMEF. SEMCAD. 2007;3(2):9-56.

9. Rosendal M, Olde Hartman TC, Aamland A, van der Horst H, Lucassen P, Budtz-Lilly A, et al. "Medically unexplained" symptoms and symptom disorders in primary care: prognosis-based recognition and classification. BMC Fam Pract. 2017;18(1):18. http://dx.doi. org/10.1186/s12875-017-0592-6 
10. Steinbrecher N, Koerber S, Frieser D, Hiller W. The prevalence of medically unexplained symptoms in primary care. Psychosomatics. 2011;52(3):263-71. http://dx.doi.org/10.1016/j.psym.2011.01.007

11. Tófoli LF, Andrade LH, Fortes S. Somatization in Latin America: a review of the classification of somatoform disorders, functional syndromes and medically unexplained symptoms. Rev Bras Psiquiatr. 2011;33 Suppl 1:S59-80.

12. Melzack R. Gate control theory: On the evolution of pain concepts. Pain Forum. 1996;5(2):128-38. http://dx.doi.org/10.1016/S10823174(96)80050-X

13. Bener A, Verjee M, Dafeeah EE, Falah O, Al-Juhaishi T, Schlogl J, et al. Psychological factors: anxiety, depression, and somatization symptoms in low back pain patients. J Pain Res. 2013;6:95-101. http://dx.doi.org/10.2147/JPR.S40740

14. Darlow B, Fullen BM, Dean S, Hurley DA, Baxter GD, Dowell A. The association between health care professional attitudes and beliefs and the attitudes and beliefs, clinical management, and outcomes of patients with low back pain: a systematic review. Eur J Pain. 2012;16(1):317. http://dx.doi.org/10.1016/j.ejpain.2011.06.006

15. Gatchel RJ, Peng YB, Peters ML, Fuchs PN, Turk DC. The biopsychosocial approach to chronic pain: scientific advances and future directions. Psychol Bull. 2007;133(4):581-624. http://dx.doi.org/10.1037/0033-2909.133.4.581

16. Koerich MHAL. O cuidado das pessoas com dor lombar crônica e o modelo de cuidado na atenção básica à saúde [Tese de doutorado]. Florianópolis: Universidade Federal de Santa Catarina; 2016. 380 p.

17. Brasil. Ministério da Saúde. Secretaria de Atenção à Saúde. Departamento de Atenção Básica. Acolhimento à demanda espontânea: queixas mais comuns na Atenção Básica. Brasília: Ministério da Saúde; 2013.

18. Mendes EV. A atenção primária à saúde no SUS. Fortaleza: Escola de Saúde Pública do Ceará; 2002. 89 p.

19. Goncalves DA, Fortes S, Campos M, Ballester D, Portugal FB, Tófoli LF, et al. Evaluation of a mental health training intervention for multidisciplinary teams in primary care in Brazil: a pre- and posttest study. Gen Hosp Psychiatry. 2013;35(3):304-8. http://dx.doi. org/10.1016/j.genhosppsych.2013.01.003

20. Gonçalves DA, Mari Jde J, Bower P, Gask L, Dowrick C, Tófoli LF, et al. Brazilian multicentre study of common mental disorders in primary care: rates and related social and demographic factors. Cad Saude Publica. 2014;30(3):623-32. http://dx.doi.org/10.1590/0102$311 \times 00158412$

21. Fortes S, Villano LA, Lopes CS. Nosological profile and prevalence of common mental disorders of patients seen at the Family Health Program (FHP) units in Petrópolis, Rio de Janeiro. Rev Bras Psiquiatr. 2008;30(1):32-7. http://dx.doi.org/10.1590/S151644462006005000066

22. Fortes S, Lopes CS, Villano LAB, Campos MR, Gonçalves DA, Mari JJ. Transtornos mentais comuns em Petrópolis-RJ: um desafio para a integração da saúde mental com a estratégia de saúde da família. Rev Bras Psiquiatr. 2011;33(2):150-6. http://dx.doi.org/10.1590/ S1516-44462011000200010

23. Botega NJ, Bio MR, Zomignani MA, Garcia Jr C, Pereira WAB. Transtornos do humor em enfermaria de clínica médica e validação de escala de medida (HAD) de ansiedade e depressão. Rev Saúde Pública. 1995;29(5):359-63. http://dx.doi.org/10.1590/S003489101995000500004

24. Fabião C, Costa E Silva C, Fleming M, Barbosa A. SOMS-2: translation into portuguese of the screening for Somatoform Disorders. Acta Med Port. 2008;21(3):241-6.

25. Mari JJ, Williams P. A comparison of the validity of two psychiatric screening questionnaires (GHQ-12 and SRQ-20) in Brazil, using Relative Operating Characteristic (ROC) analysis. Psychol Med. 1985;15(3):651-9. http://dx.doi.org/10.1017/S0033291700031500

26. Villano LA. Nanhay ALG, Moraes LR, Costa e Silva JA. Results from the Rio de Janeiro center. In: Ütbs N, ed. Mental IIIness in General Health Care: an International Study. Chichester: John Wiley \& Sons; 1995.

27. Villano LA. Problemas psicológicos e morbidade psiquiátrica em serviços de saúde não-psiquiátricos: o ambulatório de clínica geral [Tese de doutorado]. São Paulo: UNIFESP; 1998.

28. Gili M, Bauzá N, Vives M, Moreno S, Magallón R, Roca M. Validación de la versión española de la escala Screening for Somatoform Symptoms-2 para la evaluación de síntomas somáticos en Atención Primaria. Aten Primaria. 2014;47(5):273-8. http://dx.doi.org/10.1016/j. aprim.2014.07.001

29. Manchikanti L, Pampati V, Fellows B, Beyer CD, Damron KS, Barnhill RC, et al. Characteristics of chronic low back pain in patients in an interventional pain management setting: a prospective evaluation. Pain Physician. 2001;4(2):131-42. 
30. Reme SE, Tangen T, Moe T, Eriksen HR. Prevalence of psychiatric disorders in sick listed chronic low back pain patients. Eur J Pain. 2011;15(10):1075-80. http://dx.doi.org/10.1016/j.ejpain.2011.04.012

31. Reme SE, Lie SA, Eriksen HR. Are 2 questions enough to screen for depression and anxiety in patients with chronic low back pain? Spine (Phila Pa 1976). 2014;39(7):E455-62. http://dx.doi.org/10.1097/BRS.0000000000000214

32. Mirzamani-Bafghi SM, Sadidi A, Sahrai J. Psychological aspects of low back pain. Arch Iran Med. [Internet]. 2003 [acesso 2016 Jul 12]; 6(2):91-4. Available from: http://www.ams.ac.ir.htm

33. Pinheiro MB, Ferreira ML, Refshauge K, Ordoñana JR, Machado GC, Prado LR, et al. Symptoms of Depression and Risk of New Episodes of Low Back Pain: A Systematic Review and Meta-Analysis. Arthritis Care Res (Hoboken). 2015;67(11):1591-603. http://dx.doi.org/10.1002/ acr.22619

34.Ponte C. Lombalgia em cuidados de saúde primários: Sua relação com características sociodemográficas. Rev Port Clin Geral. 2005;21(3):259-67.

35. Bener A, El-Rufaie OF, Kamran S, Georgievski AB, Farooq A, Rysavy M. Disability, depression and somatization in a low back pain population. APLAR J Rheumatol. 2006;9(3):257-63. http://dx.doi.org/10.1111/j.1479-8077.2006.00210.x

36. Flora FAM. As origens históricas da Clínica e suas implicações sobre a abordagem dos problemas psicológicos na prática médica. Rev Bras Med Fam Comunidade. 2006;2(7):203-16. http://dx.doi.org/10.5712/rbmfc2(7)56

37. Balint M. O médico, seu paciente e a doença. Rio de Janeiro: Atheneu; 1984 p.11.

38. Vescovi RGL, Souza LGS, Avellar LZ. Usuários poliqueixosos: representações sociais construídas por médicos da atenção primária à saúde. Psicol Est. 2015;20(1):71-82. http://dx.doi.org/10.4025/psicolestud.v20i1.25519

39. Campos GWS. A mediação entre conhecimento e práticas sociais: a racionalidade da tecnologia leve, das práxis e da arte. Ciênc Saúde Coletiva. 2011;16(7):3033-40. http://dx.doi.org/10.1590/S1413-81232011000800002

40. Fortes S, Tófoli LF, Chazan LF, Ballester D. Queixas Somáticas sem Explicação Médica. In: Duncan BB, Schimidt MI, Giugliani ERJ, Duncan MS, Giugliani C, orgs. Medicina ambulatorial: condutas de atenção primária baseadas em evidências. Porto Alegre: Artmed; 2013. p.1138-47.

41. Ferreira MS, Navega MT. Efeitos de um programa de orientação para adultos com lombalgia. Acta Ortop Bras. 2010;18(3):127-31. http://dx.doi.org/10.1590/S1413-78522010000300002

42. Borges RG, Vieira A, Noll M, Bartz PT, Candotti CT. Efeitos da participação em um Grupo de Coluna sobre as dores musculoesqueléticas, qualidade de vida e funcionalidade dos usuários de uma Unidade Básica de Saúde de Porto Alegre - Brasil. Motriz. 2011 ;17(4):719-27.

43. Bartz PT, Bueno AF, Vieira A. Grupo da coluna na atenção básica. Cad Educ Saúde Fisiot. 2015;2(3):53-65.

44. Lancet. Low back pain. Executive Summary. [acesso 2018 Jul 20]. Disponível em: https://www.thelancet.com/series/low-back-pain

45. Hartvigsen J, Hancock MJ, Kongsted A, Louw Q, Ferreira ML, Genevay S, et al.; Lancet Low Back Pain Series Working Group. What low back pain is and why we need to pay attention. Lancet. 2018;391(10137):2356-67. http://dx.doi.org/10.1016/S0140-6736(18)30480-X

46. Foster NE, Anema JR, Cherkin D, Chou R, Cohen SP, Gross DP, et al.; Lancet Low Back Pain Series Working Group. Prevention and treatment of low back pain: evidence, challenges, and promising directions. Lancet. 2018;391(10137):2368-83. http://dx.doi.org/10.1016/ S0140-6736(18)30489-6

47. Buchbinder R, van Tulder M, Öberg B, Costa LM, Woolf A, Schoene M, et al.; Lancet Low Back Pain Series Working Group. Low back pain: a call for action. Lancet. 2018;391(10137):2384-8. http://dx.doi.org/10.1016/S0140-6736(18)30488-4

48. Chiaverini DH, org. Guia prático de matriciamento em Saúde Mental. Brasília: Ministério da Saúde, Centro de Estudos e Pesquisa em Saúde Coletiva; 2011.

49. Fonseca MLG, Guimarães MBL, Vasconcelos EM. Sofrimento difuso e transtornos mentais comuns: uma revisão bibliográfica. Rev APS. 2008;11(3):285-94.

50. Stewart M, Brown JB, Weston W, Mc Whinney IA, McWilliam CL, Freeman TR. Medicina Centrada na Pessoa: Transformando o método clínico. $2^{a}$ ed. Porto Alegre: Artmed; 2010.

51. Bonfim IG, Bastos ENE, Gois CWL, Tofoli LF. Apoio matricial em saúde mental na atenção primária à saúde: uma análise da produção científica e documental. Interface (Botucatu). 2013;17(45):287-300. http://dx.doi.org/10.1590/S1414-32832013005000012 